\title{
Comparative investigation of damage induced by diatomic and monoatomic ion implantation in silicon
}

\author{
T. Lohner ${ }^{a}$, Z. Tóth ${ }^{b} *$, M. Fried ${ }^{a}$, N.Q. Khánh ${ }^{a}$, Gen Qing Yang ${ }^{c}$, Lin Chen Lu ${ }^{c}$, \\ Shichang Zou ${ }^{c}$, L.J. Hanekamp ${ }^{d}$, A. van Silfhout ${ }^{d}$, J. Gyulai ${ }^{a}$ \\ a Joint Chair for Experimental Physics of Technical University Budapest and of KFKI Research Institute \\ for Materials Science P.O. Box. 49, H-1525 Budapest, Hungary \\ ${ }^{b}$ TUNGSRAM Co. Ltd., Bródy I. Research Center, IV., Váci út 77., H-1340 Budapest, Hungary \\ c Shanghai Institute of Metallurgy, 200050 Shanghai, China \\ ${ }^{d}$ Faculty of Applied Physics, University of Twente, P.O. Box 217, 7500 AE Enschede, The Netherlands
}

The damaging effect of mono- and diatomic phosphorus and arsenic ions implanted into silicon was investigated by spectroscopic ellipsometry (SE) and high-depth-resolution Rutherford backscattering and channeling techniques. A comparison was made between the two methods to check the capability of ellipsometry to examine the damage formed by room temperature implantation into silicon. For the analysis of the spectroscopic ellipsometry data we used the conventional method of assuming appropriate optical models and fitting the model parameters (layer thicknesses and volume fractions of the amorphous silicon component in the layers) by linear regression. The depth dependence of the damage was determined by both methods. It was revealed that $\mathrm{SE}$ can be used to investigate the radiation damage of semiconductors together with appropriate optical model construction which can be supported or independently checked by the channeling method. However, in case of low level damage (consisting mainly of isolated point defects) ellipsometry can give false results, ovcrcstimating the damage using inappropriate dielectric functions. In that case checking by other methods like channeling is desirable.

\section{Introduction}

The amount of defects and lattice disorder during ion implantation mostly depends on the energy deposited by the impinging species and the secondary knocked-on particles in the cascade volume. A very simple way of varying the deposited energy in the projected range is using molecular or polyatomic ion beams as an implant, and many experiments were carried out in the past comparing the effects caused by these implant with the effects caused by monoatomic ones [1-3]. These investigations conventionally were made by RBS and channeling techniques or electron microscopy. As a comparative method we examined the damage structure by spectroscopic ellipsometry (SE). The defects created in crystalline materials alter the complex dielectric function (refractive index). SE is a sensitive, rapid and nondestructive method for measuring the optical parameters of a material [4]. It is an indircct technique, so to get damage profiles from SE spectra one must have a proper multilayer optical model [5]. This multilayer model can arise from theo-

\footnotetext{
* Corresponding author, phone. +3611691466 , fax +361 1691965.
}

retical considerations. SE is very sensitive to changes in optical parameters and it is very useful in case of thin layers below the depth resolution of RBS. It is more advantageous if the layer is very close to the surface because of high surface sensitivity of SE. This is the situation with surface disordering caused by light ion bombardment of silicon or applying high dose. Our aim with this experiment was to study the differences between the damage profiles caused by mono- and diatomic implants and comparing the results with the RBS and channeling ones.

\section{Experimental}

Carefully cleaned (20\% HF dip followed by successive DI water rinse), $10 \Omega \mathrm{cm}$, p type silicon of $\langle 100\rangle$ orientation were implanted with $\mathrm{P}^{+}$and $\mathrm{P}_{2}^{+}$ions with dose $1 \times 10^{14}$ atoms $/ \mathrm{cm}^{2}$ at 25 and $45 \mathrm{keV}$ and $5 \times 10^{13}$ atoms $/ \mathrm{cm}^{2}$ at 50 and $90 \mathrm{keV} ; \mathrm{As}^{+}$and $\mathrm{As}_{2}^{+}$ions with $2 \times 10^{14}$ atoms $/ \mathrm{cm}^{2}$ at $30 \mathrm{keV}$ and $10^{14}$ atoms $/ \mathrm{cm}^{2}$ at $60 \mathrm{keV}$; so in each mono/diatomic implant couples the total number of implanted atoms and the implantation energy/atoms were the same. The implantations were made at room temperature. The fluences were chosen 
to be below or near the amorphization threshold. To avoid channeling effects during implantation a deliberate misalignment of the wafer normal of $7^{\circ}$ was used.

The ellipsometric measurements were made using a home-made rotating analyser ellipsometer in the 300 $650 \mathrm{~nm}$ region at Twente University.

The high depth resolution RBS/channeling measurements were made in KFKI using $1.5 \mathrm{MeV}$ He ions at $\Theta=97^{\circ}$ scattering angle (with a glancing exit angle of $7^{\circ}$ to the surface). In this case the depth resolution is $3 \mathrm{~nm}[6]$.

\section{Results and discussion}

For the evaluation of ellipsometric data we used the optical model, consisting of a native oxide layer, a totally amorphous layer and a partly damaged region consisting of a stack of layers with fixed and equal thicknesses with two coupled half Gaussian shaped damage profiles (Fig. 1). To get damage profile from ellipsometric spectra we used linear regression analysis (multiparameter fitting). The fitting parameters were the thickness of the oxide and amorphous layers, the place and height of the maximum of the damage profile and the half-widths of the full maxima of the two Gaussians [5]. The optical parameters of the damaged layers were calculated using the Bruggeman effective medium approximation. The results of the fittings are shown in the Figs. 2-4. As it can be seen the agreement between the measured and calculated spectra is really good. The goodness of the fit is estimated by the unbiased estimator:

$$
\begin{aligned}
\sigma= & \frac{1}{2 N-p-1}\left\{\sum _ { j = 1 } ^ { N } \left[\left(\cos \Delta_{j}^{\exp }-\cos \Delta_{j}^{\text {calc }}\right)^{2}\right.\right. \\
& \left.\left.+\left(\tan \Psi_{j}^{\exp }-\tan \Psi_{j}^{\text {cal }}\right)^{2}\right]\right\}^{1 / 2},
\end{aligned}
$$

where $N$ is the number of wavelengths and $p$ is the number of fitted parameters. The results are shown in Table 1.

For the determination of the damage profile from channeling spectra we used the computer code RBX [7] which can handle RBS and channeling spectra as well. The thickness of the oxide was determined from the area of the oxygen peak. The channeling spectra were evaluated in the usual way using the virgin and random spectra as well after subtracting the oxide peak. To evaluate the enhanced surface peak we extrapolated the buried disorder peak onto the surface from the damage distribution as a function of depth as a background for surface amorphous layer and after subtracting this background we determined the total number of displaced atoms under the native oxide. The

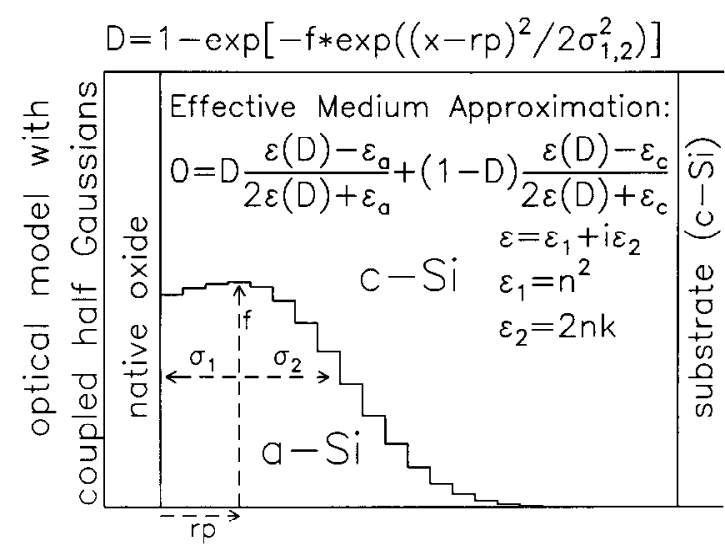

Fig. 1. Optical model for SE evaluation.

damage profile results are shown together with the ellipsometric ones in the insets of Figs. 2-4.

The agreement between the damage distribution evaluated by the two methods is very good in case of the $25 / 50 \mathrm{keV} \mathrm{P}+/ \mathrm{P}_{2}^{+}$couple, but poor in the other two cases. However, the shape of the damage profiles obtained by the two methods is similar in each case. It shows that the applied optical model is appropriate
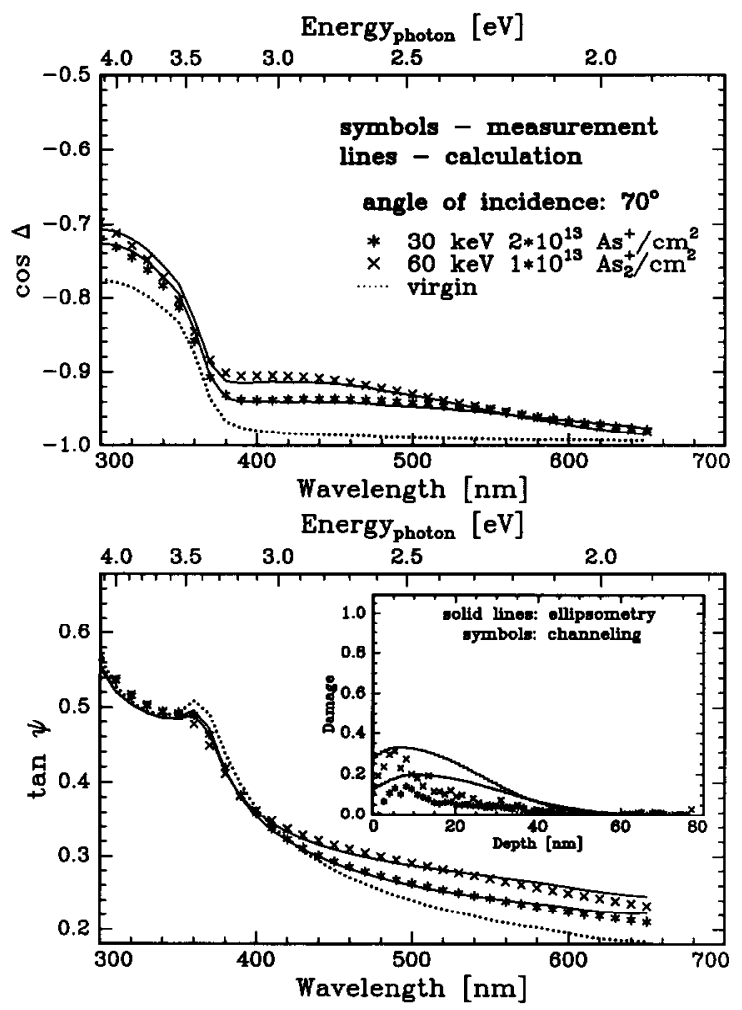

Fig. 2. Results of SE fitting for As implanted samples. The inset shows the deduced damage profiles from SE and RBS. 


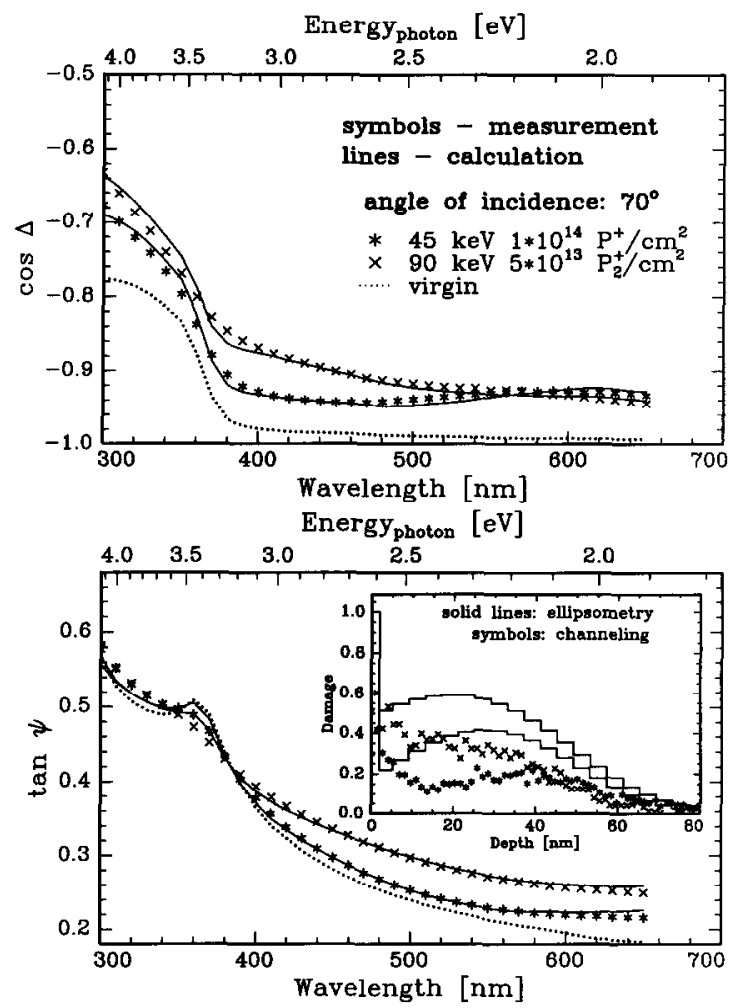

Fig. 3. Results of SE fitting for higher energy $P$ implanted samples. The inset shows the deduced damage profiles from SE and RBS.

and SE results are qualitatively good about the defect distribution, but for more precise results more adequate assumptions and models are needed.

The damage level calculated from ellipsometry was always higher or equal to that of the channeling ones. The difference between the two results can be caused by the character of the damage. Using light ions, high implantation energies or low doses the damaged region contains mainly point defects, i.e. displaced atoms. By the channeling technique we can detect these individ-

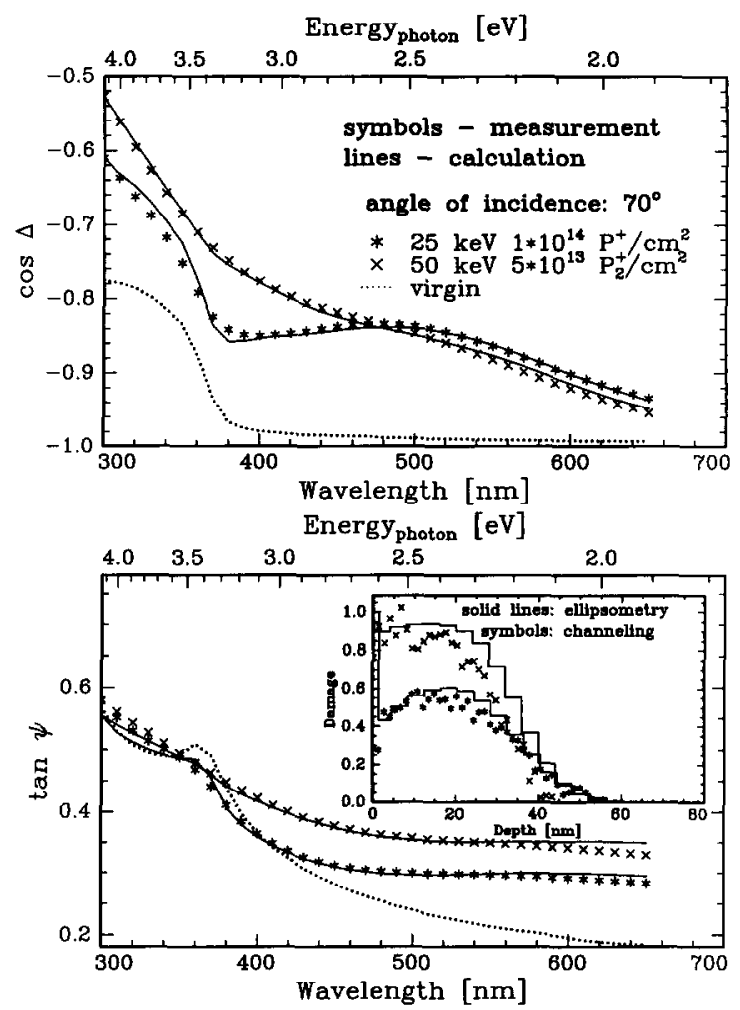

Fig. 4. Results of SE fitting for lower energy $P$ implanted samples. The inset shows the deduced damage profiles from SE and RBS.

ual point defects with high accuracy, i.e. channeling measures the number of displaced atoms. In case of ellipsometry we used the complex dielectric function of the a-Si as reference in the optical model [8]. The point defects seem to change the dielectric function in a bigger volume than their atomic volume. Consequently, SE senses the volume of the optically changed (stressed) lattice.

Further measurements to examine the role of the point defects in changing the optical parameters are

Table 1

Implantation conditions together with the results of the SE fitting procedure and RBS/channeling analysis

\begin{tabular}{|c|c|c|c|c|c|c|c|}
\hline \multirow[t]{2}{*}{ Ion } & \multirow[t]{2}{*}{ Energy $[\mathrm{keV}]$} & \multirow[t]{2}{*}{ Dose $\left[\mathrm{i} / \mathrm{cm}^{2}\right]$} & \multicolumn{2}{|c|}{ Damage [\%] } & \multicolumn{2}{|c|}{$d_{\text {amorph }}[\mathrm{nm}]$} & \multirow[t]{2}{*}{$\sigma\left[\times 10^{-3}\right]$} \\
\hline & & & $\overline{S E}$ & RBS & $\overline{\mathrm{SE}}$ & RBS & \\
\hline $\mathrm{As}^{+}$ & 30 & $2 \times 10^{13}$ & 0.2 & 0.15 & - & - & 0.8 \\
\hline $\mathrm{As}^{+}$ & 60 & $1 \times 10^{13}$ & 0.33 & 0.31 & - & - & 1.03 \\
\hline $\mathbf{P}^{+}$ & 45 & $1 \times 10^{14}$ & 0.41 & 0.24 & $1.1 \pm 0.2$ & $1.2 \pm 0.3$ & 0.96 \\
\hline $\mathbf{P}_{2}^{+}$ & 90 & $5 \times 10^{13}$ & 0.59 & 0.41 & $1.2 \pm 0.2$ & $1.6 \pm 0.3$ & 1.07 \\
\hline $\mathbf{P}^{+}$ & 25 & $1 \times 10^{14}$ & 0.60 & 0.58 & $0.5 \pm 0.1$ & - & 1.19 \\
\hline $\mathbf{P}_{2}^{+}$ & 50 & $5 \times 10^{13}$ & 0.94 & 0.90 & - & - & 1.02 \\
\hline
\end{tabular}


needed. Nevertheless, in case of high or even total amorphization these differences vanish as it was experienced in the case of $25 / 50 \mathrm{keV} P$ implants, where either more than $50 \%$ or total amorphization occurred. When only the thickness of the damaged layer is the question, ellipsometry can give the information very easily and quickly.

The thicknesses of the amorphous surface layers are summarised in Table 1 as well. The two methods give similar results, but the accuracy is better in case of SE.

\section{Conclusion}

It was revealed that spectroscopic ellipsometry can be used for investigating the radiation damage of semiconductors together with an appropriate optical model construction, which can be supported or independently checked by the channeling method. The sensitivity of the ellipsometric method is very good, comparable to that of channeling, but its accuracy can even be better than the other one. Nevertheless, in case of light ions, low dose and high energy, where the damage is low and damage consists of isolated point defects, ellipsometry can overestimate the damage level. In that case the checking by other methods like channeling is desirable.

\section{Acknowledgements}

Partial support from OTKA Grants (no. 3265 and no. F4378) is greatly appreciated.

\section{References}

[1] D.A. Thompson, R.S. Walker and J.A. Davies, Radiat. Eff. 32 (1977) 135.

[2] L.M. Howe and M.H. Rainville, Nucl. Instr. and Meth. B 182/183 (1981) 143.

[3] G.Q. Yang, N.Q. Khanh, M. Fried, E. Kotai, V. Schiller, L.C. Lu, J. Gyulai and S. Zou, Radiat. Eff. 115 (1991) 183.

[4] R.M.A. Azzam and N.M. Bashara, Ellipsometry and Polarized Light (North-Holland, Amsterdam, 1977).

[5] M. Fried, T. Lohner, W.A.M. Aarnink, L.J. Hanekamp and A. van Silfhout, J. Appl. Phys. 71 (1992) 2385.

[6] G. Mezey, T. Nagy, J. Gyulai, E. Kotai, A. Manuaba and T. Lohner, Proc. Int. Conf. on Ion Implantation on Semiconductors, eds. F. Chernow, J.A. Borders and D.K. Brice (Plenum, New York, 1977) 49.

[7] E. Kótai, these Proceedings (11th Int. Conf. on Ion Beam Analysis, Balatonfüred, Hungary, 1993) Nucl. Instr. and Meth. B 85 (1994) 588.

[8] M. Fried, T. Lohner, W.A.M. Aarnink, L.J. Hanekamp and A. van Silfhout, J. Appl. Phys. 71 (1992) 5260. 